\title{
NGC 4622 versus NGC 3081 Disks: Encounter versus Intrinsic Rings
}

\author{
Tarsh Freeman \\ Bevill State, Alabama, USA \\ Gene Byrd \\ University of Alabama Tuscaloosa, Alabama, USA \\ Derrick Ousley \\ University of Alabama Tuscaloosa, Alabama, USA
}

\begin{abstract}
.
Star formation processes in galaxies are far from being understood. Resonance rings of star formation are a common phenomenon of earlytype barred spiral galaxies. Most rings are regions of enhanced star formation via gas cloud collisions near resonances, under the action of a gravitational bar perturbation. A small number of rings may form in response to a mild tidal interaction with a nearby companion. In either case, resonance rings are natural dynamical experiments in star formation stellar association time evolution. We study star formation in two galaxies where resonance rings probably have these two different modes of origin. NGC 3081 has four bi-symmetric star-forming rings that are probably connected to bar-driven gas dynamics, while NGC 4622 is a nonbarred spiral where a strong one-sided ring may be connected to a past tidal interaction. We model the young clusters in the rings as two-fold and one-fold periodic orbits, respectively. We show that age indicators, $H \alpha$ emission and the color index $B-I$, vary with position angle around the rings in a two-fold and one-fold sense as predicted by our models. The success of these models shows that future high resolution observations can be used to separate the individual associations from the underlying disk light so as to study their evolution and luminosity function.
\end{abstract}

\section{Resonance Ring Types and Locations}

Three ring types coexist in NGC 3081 (see Figure 1a). Nuclear resonance rings are the smallest and lie well inside the bar at $\approx 750 \mathrm{pc}$ radius (see center of Figure 1a). Inner rings encircle the ends of the bar at $\approx 5 \mathrm{kpc}$ and are usually elongated along the bar axis (see the pointed oval in the middle of the Figure 1a image). Outer rings are more diffuse features about twice the size of an inner ring and which are elongated perpendicular to bars as in Figure 1a. The NGC 4622 ring is a new one-sided type (Figure $1 \mathrm{~b}$ with the arms spiraling into it). 

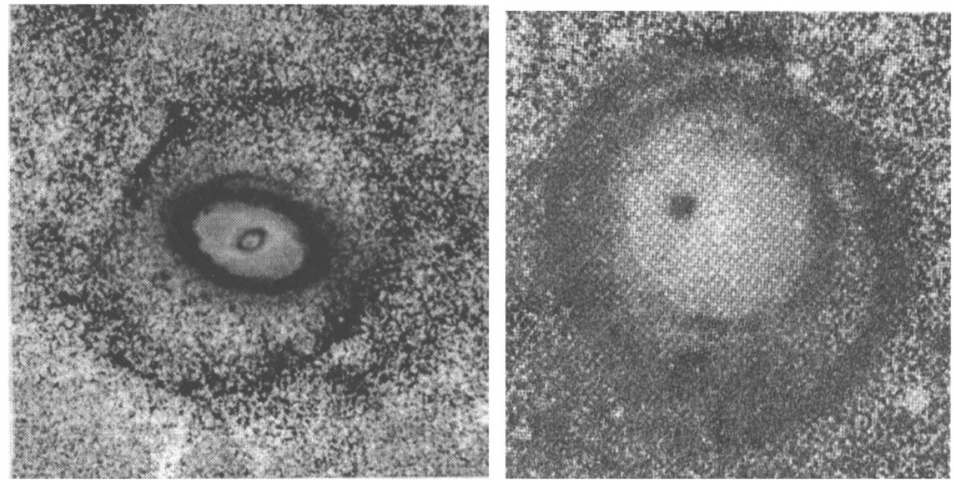

Figure 1. $\quad B-I$ Color Index CCD images of NGC 3081 (a, left panel) and NGC 4622 (b, right panel). Star forming regions appear dark.

Rings are located near resonances. Denote the circular orbital angular velocity by $\Omega$ and the radial epicyclic frequency by $\kappa$. An inner Lindblad resonance (ILR) occurs where the bar pattern speed, $\Omega_{p}=\Omega-\kappa / 2$, which in most galaxies may occur at several radii. Studies (see review by Buta and Combes 1996) have linked nuclear rings to the region between two such ILRs in barred galaxies. Inner rings have been linked to the inner 4:1 ultraharmonic resonance (UHR), where $\Omega_{p}=\Omega-\kappa / 4$. The UHR tends to be close to the corotation resonance (CR), where $\Omega_{p}=\Omega$, generally close to the bar ends. Outer rings have been linked to the outer Lindblad resonance, OLR, where $\Omega_{p}=\Omega+\kappa / 2$. These resonances are necessary but not sufficient for ring formation. A lack of gas (as in S0's) may preclude ring star formation.

In the case of a tidal interaction, a 1:1 resonance with the retrograde motion of a small companion at closest approach may lead to a lop-sided star-forming resonance ring (Byrd, Freeman, and Howard 1993). This resonance occurs where the companion at close approach creates creates a one-sided disturbance whose angular speed equals $\Omega-\kappa$ of the disk gas at the ring.

\section{NGC 3081 and NGC 4622: Two Origins of Resonance Rings}

Both NGC 3081 and NGC 4622 are very well-studied observationally (Buta and Purcell 1998; Buta, Crocker, and Byrd 1992; Scott 1995). H $\alpha$ Fabry-Perot interferometry of both galaxies reveals $\mathrm{H}$ II regions in the three innermost rings of NGC 3081 and in the ring of NGC 4622 along with blue colors around the rings, all characteristic of discrete young clusters.

Inner and outer ring clusters have not been studied theoretically and observationally. NGC 3081 and 4622 are two cases where we hope to tie the properties of the ring cluster systems to resonant dynamics. NGC 3081 has resonance rings whose distinctive shapes and alignments relative to the bar bear a strong resemblance to gaseous resonance rings in test-particle models of isolated barred spirals (e.g. Byrd et al. 1994). NGC 4622 is a nonbarred ringed spiral where the characteristics of the one-sided ring clearly point to a tidal origin. A 

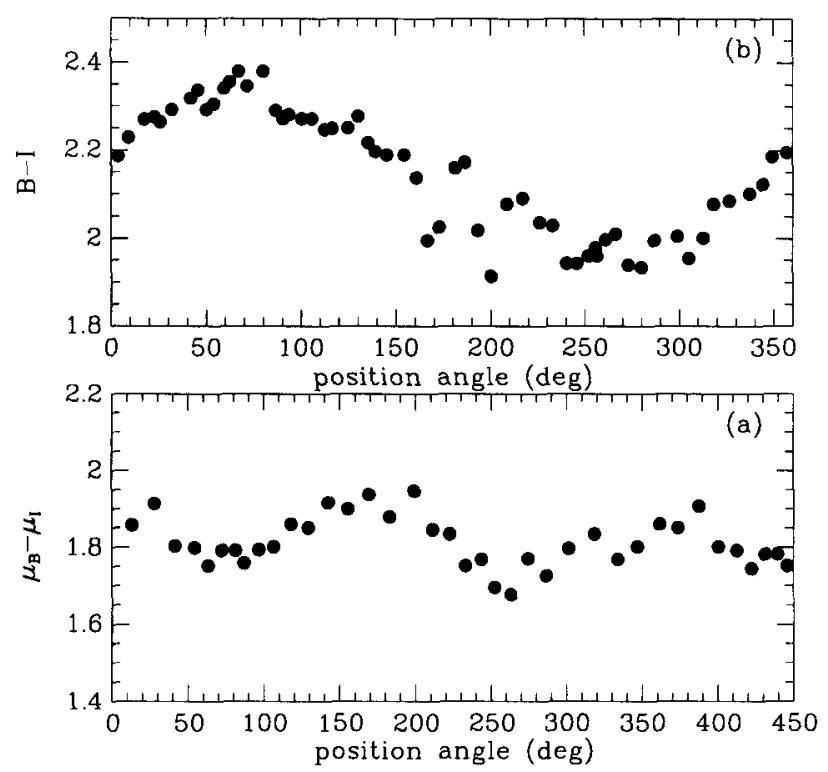

Figure 2. Color versus position angle around the inner rings of NGC 3081 (a, lower panel) and NGC 4622 (b, upper panel).

small dwarf elliptical companion seen nearby may have been the intruder (Byrd, Freeman, and Howard 1993).

The distinction between the two galaxies is shown by plots of color index versus position angle. NGC 3081's inner ring (Figure 2a) shows a two-fold variation in color over $360^{\circ}$ in position angle, while NGC 4622's ring (Figure $2 b)$ shows a one-fold variation. The elongated inner ring of NGC 3081 is bluest in arcs around its two apocentrum points at position angles of $80^{\circ}$ and $260^{\circ}$ (the bar axis). There are also peaks of $\mathrm{H} \alpha$ emission from young stars at these points. The ring of NGC 4622 is bluest (and has luminous H II regions) in an arc around its single apocentrum point near position angle $270^{\circ}$.

In terms of characteristic periodic orbits lying inside CR (Contopoulos 1979): gas circulating around the rings spends more time near apocentrum than pericentrum, favoring enhanced star formation at apocentrum. We have developed analytic/computer periodic orbit models for NGC 3081 and 4622, respectively using the method of Byrd, Ousley and Dalla Piazza (1998). Figure (3a) shows our periodic orbit model of NGC 3081 where gas clouds would gently collide to form stars. Figure ( $3 \mathrm{~b}$ ) is a similar plot for NGC 4622 with the ring periodic orbit marked with a heavy solid line. The corresponding ring in the Figure 1b image has the spiral arms spiraling into it. The orbit model indicates there could be a smaller one-sided ring interior to the NGC4622 ring but there is evidently not enough gas there for star formation. 

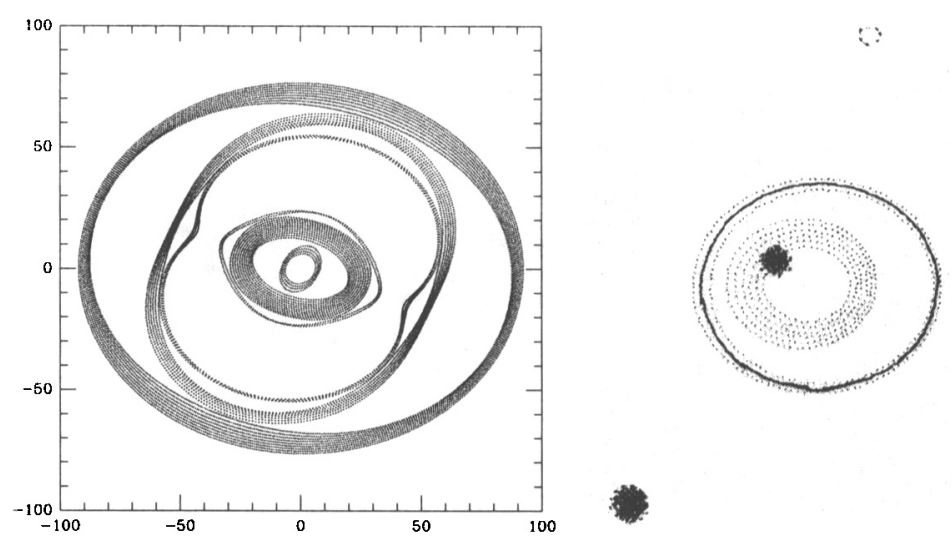

Figure 3. (3a, left panel) Periodic orbit model of NGC 3081. $\Omega_{p}=$ $4.98\left(\mathrm{~km} \mathrm{~s}^{-1}\right) /{ }^{\prime \prime}, i=31^{\circ}$. Position angles $(p a)$ of line of nodes $\Omega_{a p}=$ $100^{\circ}$ and of the bar $B_{p a}=71^{\circ}$. Circular orbital speed, $V_{0}=221$ $\mathrm{km} \mathrm{s}^{-1}$. Perturbation amplitude, $q=0.035 V_{0}^{2}$ with $q_{2}=0.95 q$ and $q_{4}=0.05 q$. (3b, right panel) NGC 4622 right. $\Omega_{p}=-3.5\left(\mathrm{~km} \mathrm{~s}^{-1}\right) /{ }^{\prime \prime}$, $i=31^{\circ} . \Omega_{p a}=70^{\circ}, B_{p a}=60^{\circ} . V_{0}=200 \mathrm{~km} \mathrm{~s}^{-1}$. One-fold $q_{1}=$ $0.007 V_{0}^{2}$ Fig. $1 \mathrm{~b}$ "stars" are added for scale.

\section{Color Variations around the Two Kinds of Rings}

Our analytic/computer models predict the two-fold and one-fold color variations seen around the rings of NGC 3081 and 4622, in particular, how the age of a cluster will vary with position angle from the apocentric point. Clusters born near the major axis of the NGC 3081 inner ring will be about $200 \mathrm{Myr}$ old when they reach the opposite side. For NGC 3081, we obtain an observational relation $B-I=1.76+0.0015 T$ where $T$ is the age in millions of years.

A similar relation can be obtained for NGC 4622 but its rotation curve and disk inclination are not so well known. The zero age point of the NGC4622 relation is $B-I=1.9$, somewhat redder than the $B-I=1.76$ NGC3081 value. NGC 4622's distance of $56 \mathrm{Mpc}$ is farther than the $33 \mathrm{Mpc}$ of NGC 3081. For both galaxies, the ring clusters are not easily separated from the background older redder disk starlight with the problem evidently being worse for NGC 4622. Our empirical color age relation above is thus far only qualitative. We hope to image NGC 3081 and NGC 4622 with better resolution so that we can remove the disk light and estimate colors for the individual ring clusters for both galaxies as a function of radius and position angle. Then the observational colors and ages can be directly compared to evolutionary synthesis color vs. age models of, e.g., Bruzual and Charlot (1996) as an observational test of the models and a study of the effects of interstellar dust on the observation of associations in other galaxies.

Acknowledgments. Support was provided by NSF grant RUI 9802918 and by the Academy of Finland. 


\section{References}

Bruzual, G. and Charlot, S. 1996, AAS CD-ROM, Vol. 7

Buta, R. and Combes, F. 1996, Fund. Cosmic Phys., 17, 95

Buta, R. and Purcell, G. 1998, AJ, 115, 484

Buta, R., Crocker, D., and Byrd, G. 1992, AJ, 103, 152

Byrd, G., Freeman, T., and Howard, S. 1993, AJ, 105, 477

Byrd, G., Rautiainen, P., Salo, H., Buta, R., and Crocker, D., 1994, AJ 108, 476.

Byrd, G., Ousley, D., and Dalla Piazza, C. 1998, MNRAS, 298, 7

Contopoulos, G. 1979, in Photometry, Kinematics, and Dynamics of Galaxies, ed. D. S. Evans, Univ, of Texas Press, p. 425

Scott, J. 1995, Master's Thesis, Univ. of Alabama 